\title{
FGFR3-TACC3: a novel gene fusion in malignant melanoma
}

\section{Jiyun Lee ${ }^{1}$, Jeeyun Lee ${ }^{1}$, Sang Duk Hong ${ }^{2}$, Kee-Taek Jang ${ }^{3}$, Su Jin Lee ${ }^{1}$}

${ }^{1}$ Division of Hematology-Oncology, Department of Medicine, Samsung Medical Center, Sungkyunkwan University School of Medicine, Seoul, Korea

${ }^{2}$ Department of Otorhinolaryngology-Head and Neck Surgery, Samsung Medical Center, Sungkyunkwan University School of Medicine, Seoul, Korea

${ }^{3}$ Department of Pathology and Translational Genomics, Samsung Medical Center, Sungkyunkwan University School of Medicine, Seoul, Korea

Received: April 262018

Revised: May 182018

Accepted: June 12018

Corresponding author:

Su Jin Lee

Division of Hematology-

Oncology, Department of

Medicine, Samsung Medical

Center, Sungkyunkwan

University School of Medicine,

81 Irwon-ro, Gangnam-gu,

Seoul 06351, Korea

Tel: +82-2-3410-3459

E-mail: ssjj.lee@samsung.com

This is an Open Access article distributed under the terms of the Creative Commons Attribution Non-Commercial License (http:// creativecommons.org/licenses/ by-nc/4.0/).

\section{ABSTRACT}

Oncogenic gene fusions have been identified in many cancers, and many serve as druggable targets for therapy. Fibroblast growth factor receptor (FGFR) gene aberration is known to be associated with tumor progression and resistance to anticancer therapy. Here we report the first case of malignant melanoma harboring a FGFR3-TACC3 (transforming acidic coiled-coil containing protein 3 ) fusion, which appears to be a promising potential therapeutic target.

Keywords: FGFR3-TACC3; Malignant melanoma; Novel fusion

\section{INTRODUCTION}

Over the past decade, palliative treatment for melanoma has changed dramatically. The discovery of the oncogenic point mutation BRAF V600 and subsequent development of BRAF inhibitors and mitogen-activated protein kinase kinase (MEK) inhibitors have prolonged patient survival [1,2]. Imatinib or nilotinib for KIT mutated melanoma has shown promising response rates [3,4]. However, there are still many melanoma patients that do not have the option of targeted therapy, and the need for identification of a new therapeutic target is ever growing.

The fibroblast growth factor receptor (FGFR) family consists of four subtypes of transmembrane tyrosine kinase receptors (FGFR1-4) that play important roles in cell growth, differentiation, and angiogenesis [5]. Aberrant signaling through FGFR can occur through overexpression of receptors, ligands, and activating mutations of FGFR-containing translocations. FGFR gene fusions with multiple partners in a wide spectrum of tumors have been described. FGFR3transforming acidic coiled-coil containing protein 3 (TACC3) fusions were first reported in glioblastoma and urothelial tumors [6,7]. This fusion proved to be an important therapeutic target in solid tumors and has supported the clinical development of several FGFR inhibitors and anti-FGFR drug conjugate antibodies [8]. Herein, we describe the first case of melanoma harbor- 
PRECISION AND FUTURE MIEDICINE

FGFR3-TACC3: A novel gene fusion in malignant melanoma

ing a FGFR3-TACC3 fusion, revealed by a next-generation sequencing (NGS) assay.

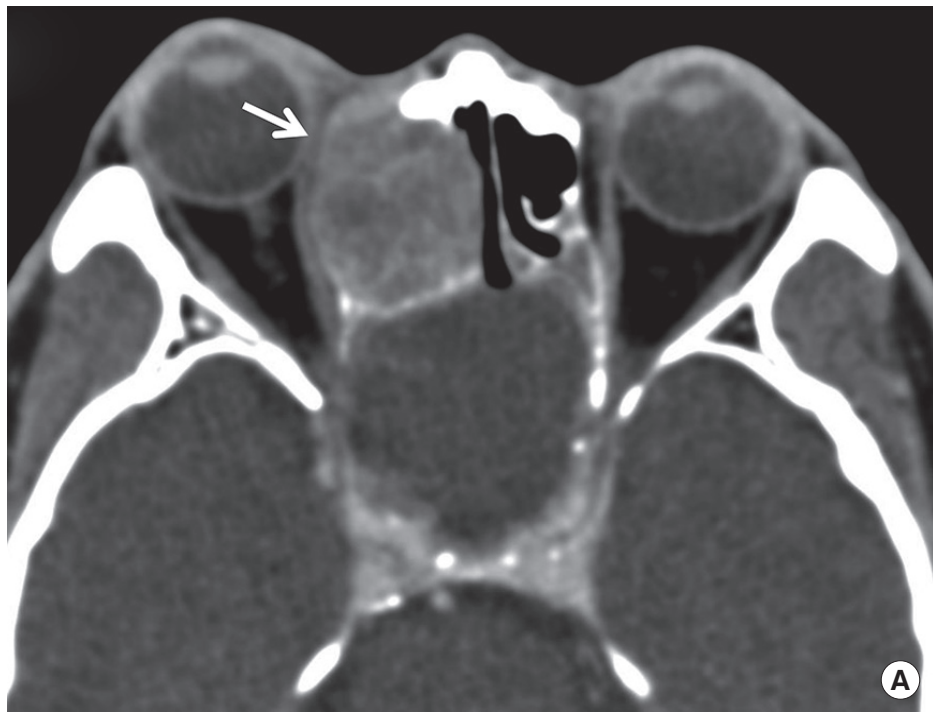

\section{CASE REPORT}

A 66-year-old male presented to a clinic with blurred vision

Fig. 1. Orbit computed tomography showing (A) a mass occupying the right ethmoid sinus and nasal cavity, (B) causing bony destruction at the skull base.
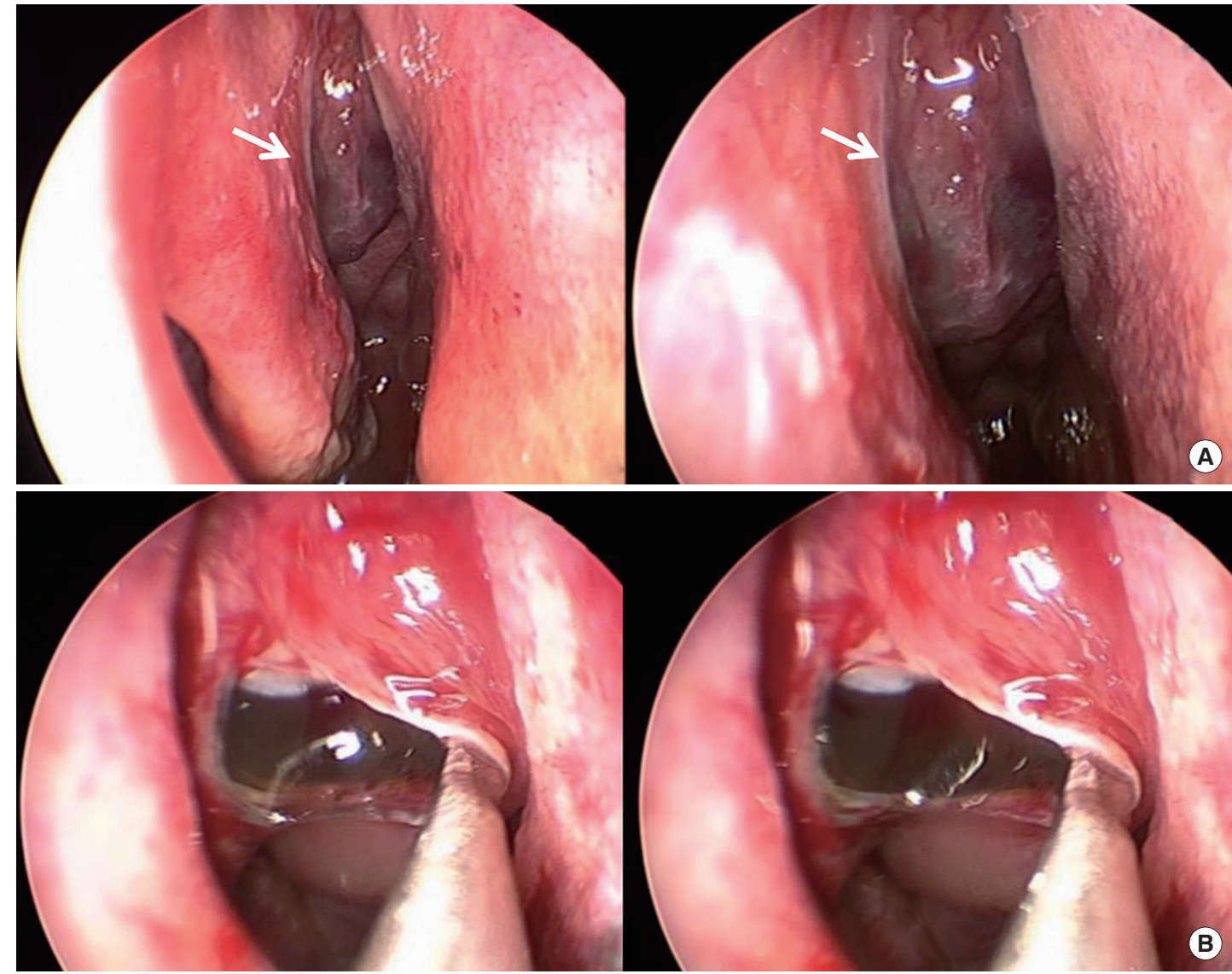

Fig. 2. Endoscopic examination revealed (A) a mass (arrows) between the right middle turbinate and nasal septum. (B) Serous discharge was drained upon biopsy. 
and right ocular swelling. An orbital computed tomography (CT) scan showed a mass with heterogeneous enhancement, occupying the right ethmoid sinus and nasal cavity, extending to the right medial orbital wall and skull base, causing

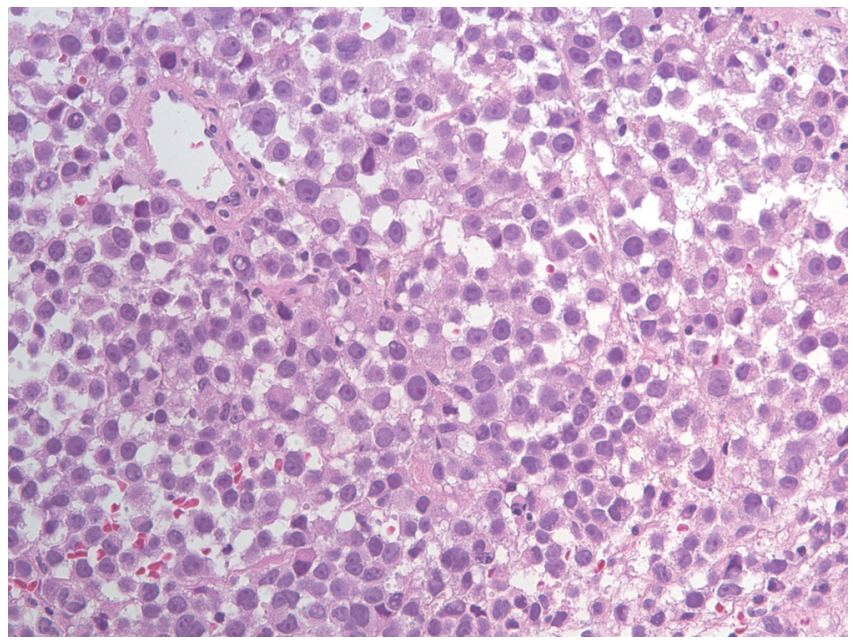

Fig. 3. High magnification of resected specimen disclosed diffuse large cell nest with prominent nucleoli and frequent plasmacytoid feature (H\&E stain, $\times 400)$. bone destruction (Fig. 1).

Endoscopic examination revealed a protruding mass with mucosal color change between the right middle turbinate and nasal septum. Serous discharge was drained upon biopsy via endoscopic sinus surgery (Fig. 2). The histopathologic finding showed diffuse large cell nest with prominent nucleoli and frequent plasmacytoid feature (Fig. 3) and positive immunoreactivity for S100 protein and HMB-45, which were consistent with malignant melanoma. The patient was referred to our clinic for systemic treatment in November 2017. Routine hematological and biochemical investigations were all within normal limits. Abdominal CT scan showed multiple metastatic masses in the perihepatic area and pelvic cavity (Fig. 4). Brain $\mathrm{CT}$ showed no definite evidence of brain metastasis. The patient started on pembrolizumab as a first-line chemotherapy, but experienced rapidly progressive disease and death due to cancer progression. A FGFR3-TACC3 fusion was identified in the tumor using a next-generation sequencing (NGS) platform (Oncomine Comprehensive Assay v3, ThermoFisher Scientific, Waltham, MA, USA; www.thermofisher.com).
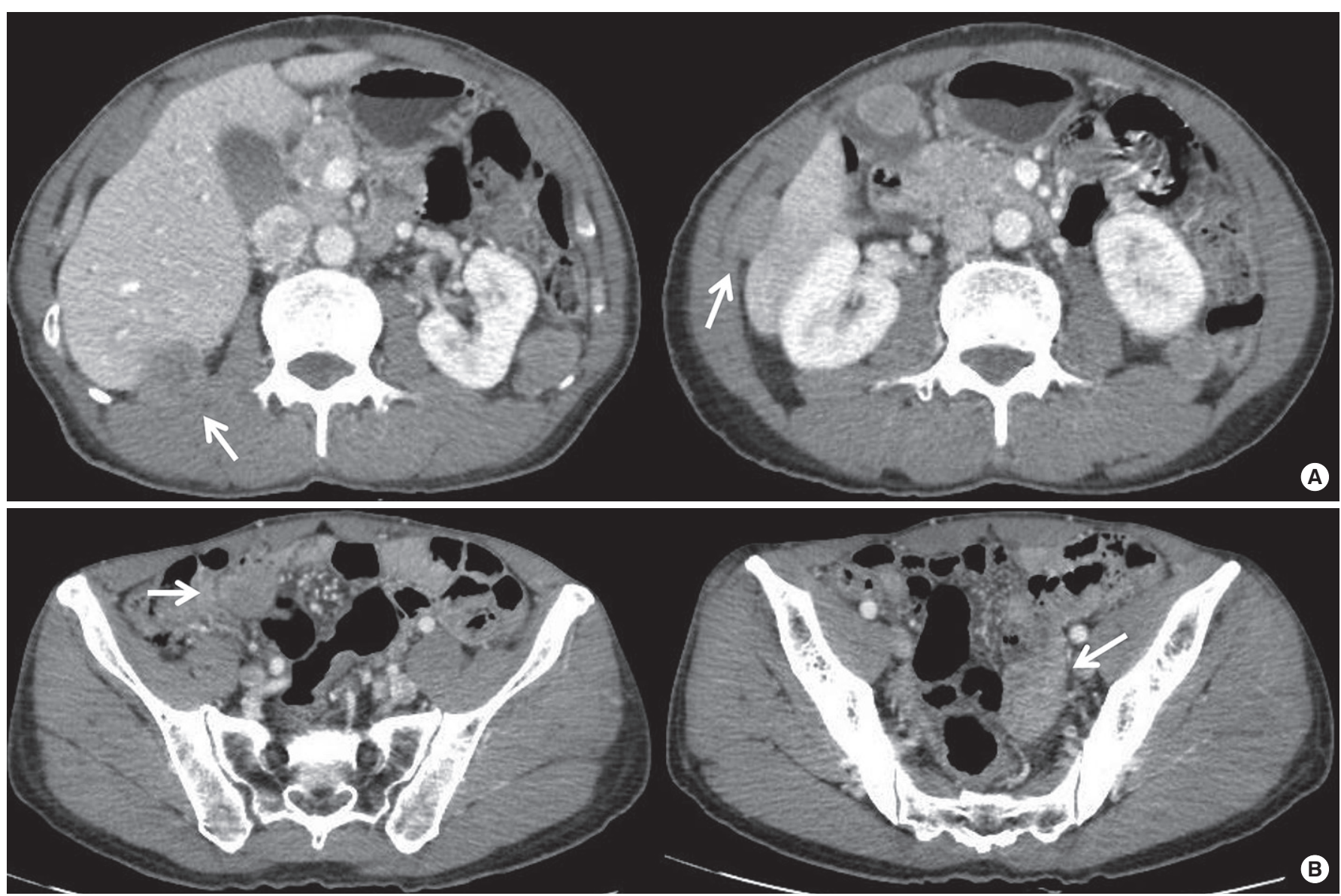

Fig. 4. Abdominal computed tomography showing multiple metastases (arrows) in the (A) perihepatic area and (B) pelvic cavity. 


\section{DISCUSSION}

The genetic aberrations most commonly found in malignant melanoma are BRAF, NRAS, and KIT mutations. BRAF mutations have been reported in $41 \%$ to $55 \%$ of metastatic melanoma. Most BRAF-activating mutations occurring in melanomas are at residue V600, usually V600E but occasionally V600K or other substitutions [9]. Inhibitors of MEK, a signaling molecule downstream of $B R A F$, potentiate the clinical activity of $B R A F$ inhibitors in melanomas with BRAF V600 mutations. Multiple studies have demonstrated superior efficacy of $B R A F /$ MEK inhibitors (dabrafenib/trametinib or vemurafenib/cobimetinib) combination therapy compared to BRAF monotherapy, with improved progression-free survival and response rate [1,2,10-14]. NRAS mutations, which have been reported in $15 \%$ to $20 \%$ of melanoma, are not yet druggable. KIT mutations, although rarer than BRAF mutations, are detected in $15 \%$ of mucosal and acral melanomas [15]. Studies have shown promising efficacy of imatinib and nilotinib, which are selective KIT inhibitors, for metastatic melanoma patients with KIT mutations [3,16,17]. However, a subset of patients has no targetable genetic aberrations and no chance to receive targeted therapies. Therefore, it is necessary to identify new targetable genetic aberrations in melanoma patients.

FGFRs consist of an extracellular domain encompassing three immunoglobulin-like domains (Igl, IgIl, IgIII), followed by a transmembrane domain and two tyrosine kinase sub-domains, TKI and TKII. Fibroblast growth factors (FGFs) and the FGFR signaling pathway are vital in the management of tissue repair, wound healing, and tumor angiogenesis. It is crucial that FGF activity is tightly regulated to maintain homeostatic cellular proliferation [18].

After the first report of FGFR3-TACC3 gene fusion in glioblastoma in 2012, an FGFR3-TACC3 fusion was reported in numerous solid tumors including urothelial carcinoma, nonsmall cell lung cancer, thyroid, and uterine cervical carcinoma [8]. In the FGFR3-TACC3 fusion protein, the final exon at the $\mathrm{C}$ terminus of FGFR3 is replaced by TACC3, which mediates localization to the mitotic spindle. The mitotic localization of FGFR3-TACC3 fusion protein causes subsequent chromosomal segregation defects, resulting in aneuploidy $[6,19]$. Further, a cell line study showed a 2-fold increase in tyrosine kinase phosphorylation on the FGFR3-TACC3 fusion protein compared to that of FGFR3 wild type [20]. Deregulation of the FGF-FGFR signaling axis has been implicated in oncogenesis, tumor progression, and resistance to anticancer therapy across many tumor types [19]. Along with preclinical results,
FGFR3-TACC3 fusion appears to be a promising target of inhibition. In a phase I trial of JNJ42756493, an oral pan-FGFR inhibitor, there were three out of four confirmed partial responders (two urothelial cancer, one glioblastoma) who harbored the FGFR3-TACC3 fusion. Tumor shrinkage was also seen in a patient with adrenal carcinoma with predominant FGFR3-TACC3 translocation [21]. More early-phase studies are needed. A phase I/II trial of AZD4547, an oral selective inhibitor of FGFR 1, 2, and 3 receptor tyrosine kinase, is currently being investigated in patients with recurrent glioma expressing FGFR3-TACC3 or FGFR1-TACC1 fusion (NCT02824133). A phase II trial of BGJ398, a selective pan-FGFR kinase inhibitor, has been conducted in recurrent glioblastoma patients with FGFR3-TACC3, FGFR1-TACC1 fusion, or activating mutation in FGFR1, 2 or 3 (NCT01975107).

To the best of our knowledge, this is the first report to identify an FGFR3-TACC3 fusion in malignant melanoma. This rare gene fusion event was identified in metastatic mucosal melanoma arising from nasal cavity at diagnosis and the clinical course was rapidly deteriorating despite of pembrolizumab. Given the aggressive and poor survival of mucosal melanoma, a better understanding of genetic changes is needed to lay the foundation for development of more effective therapeutic agents. This report provides proof of concept that treatment with an FGFR inhibitor can provide clinical benefit in malignant melanoma cases carrying FGFR3-TACC3 translocation, in agreement with results observed in other malignancies. Further, our finding highlights the importance of a comprehensive genomic profiling approach able to detect all classes of genomic alterations, including uncommon gene fusions, to reveal potentially targetable somatic alterations to inform new strategies for patients with limited therapeutic options.

\section{CONFLICTS OF INTEREST}

No potential conflict of interest relevant to this article was reported.

\section{REFERENCES}

1. Larkin J, Ascierto PA, Dreno B, Atkinson V, Liszkay G, Maio $M$, et al. Combined vemurafenib and cobimetinib in BRAFmutated melanoma. N Engl J Med 2014;371:1867-76.

2. Long GV, Stroyakovskiy D, Gogas H, Levchenko E, de Braud F, Larkin J, et al. Combined BRAF and MEK inhibition versus BRAF inhibition alone in melanoma. $N$ Engl $J$ Med 2014;371:1877-88. 
3. Carvajal RD, Antonescu CR, Wolchok JD, Chapman PB, Roman RA, Teitcher J, et al. KIT as a therapeutic target in metastatic melanoma. JAMA 2011;305:2327-34.

4. Lee SJ, Kim TM, Kim YJ, Jang KT, Lee HJ, Lee SN, et al. Phase II trial of nilotinib in patients with metastatic malignant melanoma harboring KIT gene aberration: a multicenter trial of Korean Cancer Study Group (UN10-06). Oncologist 2015;20:1312-9.

5. Touat M, lleana E, Postel-Vinay S, Andre F, Soria JC. Targeting FGFR signaling in cancer. Clin Cancer Res 2015;21: 2684-94.

6. Singh D, Chan JM, Zoppoli P, Niola F, Sullivan R, Castano A, et al. Transforming fusions of FGFR and TACC genes in human glioblastoma. Science 2012;337:1231-5.

7. Williams SV, Hurst CD, Knowles MA. Oncogenic FGFR3 gene fusions in bladder cancer. Hum Mol Genet 2013;22: 795-803.

8. Costa R, Carneiro BA, Taxter T, Tavora FA, Kalyan A, Pai SA, et al. FGFR3-TACC3 fusion in solid tumors: mini review. Oncotarget 2016;7:55924-38.

9. Long GV, Menzies AM, Nagrial AM, Haydu LE, Hamilton AL, Mann GJ, et al. Prognostic and clinicopathologic associations of oncogenic BRAF in metastatic melanoma. J Clin Oncol 2011;29:1239-46.

10. Robert C, Karaszewska B, Schachter J, Rutkowski P, Mackiewicz A, Stroiakovski D, et al. Improved overall survival in melanoma with combined dabrafenib and trametinib. N Engl J Med 2015;372:30-9.

11. Long GV, Stroyakovskiy D, Gogas H, Levchenko E, de Braud F, Larkin J, et al. Dabrafenib and trametinib versus dabrafenib and placebo for Val600 BRAF-mutant melanoma: a multicentre, double-blind, phase 3 randomised controlled trial. Lancet 2015;386:444-51.

12. Johnson DB, Flaherty KT, Weber JS, Infante JR, Kim KB, Kefford RF, et al. Combined BRAF (Dabrafenib) and MEK inhibition (Trametinib) in patients with BRAFV600-mutant melanoma experiencing progression with single-agent
BRAF inhibitor. J Clin Oncol 2014;32:3697-704.

13. Sanlorenzo M, Choudhry A, Vujic I, Posch C, Chong K, Johnston $\mathrm{K}$, et al. Comparative profile of cutaneous adverse events: BRAF/MEK inhibitor combination therapy versus BRAF monotherapy in melanoma. J Am Acad Dermatol 2014;71:1102-9.e1.

14. Ribas A, Gonzalez R, Pavlick A, Hamid O, Gajewski TF, Daud $A$, et al. Combination of vemurafenib and cobimetinib in patients with advanced BRAF(V600)-mutated melanoma: a phase 1b study. Lancet Oncol 2014;15:954-65.

15. Beadling C, Jacobson-Dunlop E, Hodi FS, Le C, Warrick A, Patterson J, et al. KIT gene mutations and copy number in melanoma subtypes. Clin Cancer Res 2008;14:6821-8.

16. Guo J, Si L, Kong Y, Flaherty KT, Xu X, Zhu Y, et al. Phase II, open-label, single-arm trial of imatinib mesylate in patients with metastatic melanoma harboring c-Kit mutation or amplification. J Clin Oncol 2011;29:2904-9.

17. Hodi FS, Corless CL, Giobbie-Hurder A, Fletcher JA, Zhu M, Marino-Enriquez A, et al. Imatinib for melanomas harboring mutationally activated or amplified KIT arising on mucosal, acral, and chronically sun-damaged skin. J Clin Oncol 2013;31:3182-90.

18. Hallinan N, Finn S, Cuffe S, Rafee S, O'Byrne K, Gately K. Targeting the fibroblast growth factor receptor family in cancer. Cancer Treat Rev 2016;46:51-62.

19. Babina IS, Turner NC. Advances and challenges in targeting FGFR signalling in cancer. Nat Rev Cancer 2017;17:31832.

20. Nelson KN, Meyer AN, Siari A, Campos AR, Motamedchaboki K, Donoghue DJ. Oncogenic gene fusion FGFR3-TACC3 is regulated by tyrosine phosphorylation. Mol Cancer Res 2016;14:458-69.

21. Tabernero J, Bahleda R, Dienstmann R, Infante JR, Mita A, Italiano A, et al. Phase I dose-escalation study of JNJ-427 56493, an oral pan-fibroblast growth factor receptor inhibitor, in patients with advanced solid tumors. J Clin Oncol 2015;33:3401-8. 